\title{
CircRUNX1 Drives Immune Evasion and anti-PD1 Immunotherapy Resistance in Lung Adenocarcinoma by the miR-4739/PCSK9/MHC I Axis
}

\section{Peng-Fei Zhang}

Zhongshan Hospital Fudan University

Yi-Fan Xu

Zhongshan Hospital Fudan University

Ling-Xian Zhang

Nanchang University

Jing Wu

Zhongshan Hospital Fudan University

Yue Fan ( $\square$ fan.yue@zs-hospital.sh.cn)

Zhongshan Hospital Fudan University

\section{Research}

Keywords: circRUNX1, Lung adenocarcinoma, PCSK9, Immune evasion, Resistance to anti-PD1

Posted Date: August 31st, 2021

DOl: https://doi.org/10.21203/rs.3.rs-835938/v1

License: (c) (i) This work is licensed under a Creative Commons Attribution 4.0 International License.

Read Full License 


\section{Abstract}

Background: Lung adenocarcinoma (LUAD) is the commonest pathological subtype of lung-derived malignant tumor. Blocking the immune checkpoints interaction between programmed death-1 (PD-1) and programmed death ligand 1 (PD-L1) via naturalizing PD-1 or PD-L1 monoclonal antibody is proved to be a effective therapeutic method for drive gene negative LUAD. Dysregulation of circular RNA (circRNA) is a hallmark of cancer and has a critical role in the progression of cancers, including LUAD. However, the exact role of circular RUNX family transcription factor 1 (circRUNX1, hsa_circ_0002360) in LUAD remains unknown.

Methods: The expression levels of circRUNX1, miR-4739, and proprotein convertase subtilisin/kexin type 9 (PCSK9) mRNA were examined by quantitative real-time polymerase chain reaction (qRT-PCR). PCSK9, histocompatibility complex I (MHC I), CD8, and Foxp3 protein expression was examined by immunohistochemistry $(\mathrm{IHC})$ or western blot analysis. In vivo assay was carried out to determine the biological function of circRUNX1 on LUAD immune evasion in vivo. The binding relationship between miR-4739 and circRUNX1 or PCSK9 was predicted and verified by Starbase V3.0 online databases, dualluciferase reporter assay, and RNA-pull down assay.

Results: CircRUNX1 expression was frequently increased in LUAD tissues, and forced circRUNX1 expression was associated with poor prognosis, big tumor size, advanced stage, and resistance to antiPD1 immunotherapy in LUAD patients. Functionally, circRUNX1 overexpression promoted LUAD immune evasion in vivo. In addition, our results demonstrated that circRUNX1 could sponge miR-4739, subsequently, upregulating the PCSK9 expression and promoting to LUAD immune evasion.

Conclusion: circRUNX1 promoted cancer immune evasion and resistance to anti-PD1 immunotherapy by regulate miR-4739/PCSK9/MHC I axis in LUAD. Taken together, our findings indicate that circRUNX1 may be a promising therapeutic target for improve the anti-PD1 therapy effect in LUAD patients.

\section{Background}

Co-operation of innate and adaptive immune systems is indispensable for cancer immune surveillance to eradicate cancer cells [1]. The emergence of anti-PD-1 immunotherapy for the treatment of non-small cell lung cancer (NSCLC) has effectively improved the prognosis of the patients [2]. However, anti-PD-1-based immunotherapy only benefits $30-40 \%$ of the NSCLC patients, and many of the patients will generate acquired resistance during the process of the therapy $[3,4]$. Lung adenocarcinoma (LUAD) is the most histopathological subtype of NSCLC [5]. Despite the advanced multidisciplinary treatment, the overall survival rate of LUAD patients remains frustrating. During the process of tumor progression, cancer cells have evolved complicated mechanisms to decrease major histocompatibility complex I (MHC I) expression and resistance to cytotoxic T lymphocytes-mediated immune attack [6]. Decreased $\mathrm{MHC} \mathrm{I}$ expression has been confirmed in a variety of cancers, including $\operatorname{LUAD}[7,8]$. Furthermore, decreased $\mathrm{MHC}$ I expression has been identified as a critical mechanism of resistance to anti-PD-1-based 
immunotherapy [9]. Nonetheless, the effect of decreased MHC I expression on the sensitivity of LUAD to anti-PD-1 immunotherapy has not been explored.

With the rapid development of high-throughput sequencing technology, circular RNA (circRNA) as a class of novel non-coding RNA with an important role in multiple pathophysiological processes, therefore, have been gradually become a hotspot area attracting of investigator's attention. Recently, it has been confirmed that dysregulation of circRNAs play a critical regulatory role in cancer progression and immune evasion [10-12]. For example, circFGFR1 promotes the expression of C-X-C motif chemokine receptor 4 (CXCR4) by sponging miR-381-3p and participates in the invasion, migration, and immune evasion of NSCLC [10]. To date, a large number of circRNAs have been verified to exist in body fluids, including exosomes [13]. In recent study, we have been reported that cancer cell-derived exosomal circUHRF1 upregulates the expression of hepatitis A virus cellular receptor 2 (TIM3) by sponging miR-449c-5p and contributes to immunosuppression of NK cells [12]. However, research on circRNA-induced immune evasion in lung adenocarcinoma (LUAD) has been relatively scarce despite mounting evidence indicating that they have critical roles in malignancy.

Recently, studies have reported that proprotein convertase subtilisin/kexin type 9 (PCSK9) expression is dysregulated in multiple cancers and participates in promoting cancer progression [14]. Importantly, PCSK9 can promote MHC I degradation in lysosome [15]. Above research results indicate that inhibiting PCSK9 might be a promising method to enhance treatment efficiency for cancers immunotherapy. Here, we reported a circRNA derived from the RUNX1 gene, and named circRUNX1 (hsa_circ_0002360). CircRUNX1 was verified to be significantly upregulated in LUAD. Furthermore, we confirmed that circRUNX1 plays a critical role in the immune evasion of LUAD by sponging miR-4739 to influence expression of $\mathrm{MHC}$ I molecular via PCSK9-dependent manner. The underlying biological molecular mechanisms and functions of circRUNX1 were indicate that circRUNX1 may represent a promising biomarker and a potential therapeutic target for immunotherapy in LUAD.

\section{Methods}

\section{Cell lines}

Human LUAD cell lines (PC-9, Calu-3, HCC-827, NCl-H1975, and A549) and the HEK-293 T cell line were purchased from the Shanghai Institute of Cell Biology, Chinese Academy of Sciences (Shanghai, China). All of the above cell lines were cultured in Dulbecco's modified Eagle's medium (DMEM, HyClone, Logan City, UT) supplemented with 100 units/ml penicillin, $100 \mu \mathrm{g} / \mathrm{ml}$ streptomycin (Gibco, Carlsbad, CA, USA), and $10 \%$ fetal bovine serum (FBS, Gibco, Carlsbad, CA, USA) in a humidified atmosphere containing $5 \%$ $\mathrm{CO}_{2}$ at $37^{\circ} \mathrm{C}$.

\section{Patients and Tissues}

The clinical tissues and samples were collected and confirmed by pathological examination at Second Affiliated Hospital of Nanchang University and Zhongshan Hospital of Fudan University. The tissues and 
samples were analyzed by immunohistochemistry (IHC), quantitative real-time polymerase chain reaction (qRT-PCR), and enzyme linked immunosorbent assay (ELISA). The clinicopathological information was collected from 1 January 2012 to 31 December 2019. The Ethics Committee of the Zhongshan Hospital of Fudan University provided ethical approval, and informed consent for collecting and preserving samples and documenting details was obtained from all of the patients.

IHC, qRT-PCR, western blotting, circRNA precipitation (circRIP), RNA immunoprecipitation (RIP), luciferase reporter assay, $\mathrm{CD} 8^{+} \mathrm{T}$ cell isolation, and fluorescence in situ hybridization (FISH) assays

IHC, qRT-PCR, western blot, In vivo circRNA precipitation (circRIP), RNA immunoprecipitation (RIP), luciferase reporter assay, $\mathrm{CD} 8^{+} \mathrm{T}$ cell isolation, and fluorescence in situ hybridization (FISH) assays were carried out as described in our previous studies $[10,12,16]$. The qRT-PCR primers used in this study are listed in Supplementary Table 1. The antibodies used in this study are listed in Supplementary Table 2. Knocked down and overexpressed circRUNX1 transfection experiments

Small hairpin RNAs (shRNAs) targeting the back-splicing site of the circRUNX1, circRUNX1overexpressing, and mutant circRUNX1-overexpressing lentivirus were synthesized by Genomeditech Co. (Shanghai, China). LUAD cell lines were transfected with lentivirus according to the manufacturer's instructions.

\section{Establishment of stable PCSK9-knockout cells}

CRISPR/Cas9 double-vector lentivirus was used to establish stable PCSK9-knockout NCl-H1975 cells. The lentivirus was purchased from the Genomeditech Co. and transfected as described in reference [10].

\section{Generation of humanized NSG (HuNSG) mice and xenograft models}

NSG mice aged 4-6 weeks were purchased from Shanghai Model Organisms. The animals were housed and treated in specific pathogen-free conditions. The method performed to generate the huNSG mice were previously established and described.[17] The animal experiments were carried out in accordance with the guidelines approved by the Shanghai Medical Experimental Animal Care Commission. Mice xenograft anti-PD-1 therapy study were carried out as described in our previous studies [10]. In brief, the experiments in the huNSG mice were approved by the Animal Experimentation Ethics Committee of Zhongshan hospital, Fudan University. A total of $2 \times 10^{6}$ cells (LUAD cells with or without increased circRUNX1 expression) were implanted subcutaneously in the left flank of the huNSG mice. When tumors reached a volume approximately $100 \mathrm{~mm}^{3}$, the mice were injected in the tail vein with a PD-1 monoclonal IgG antibody (Nivolumab) or its isotype control at $100 \mu \mathrm{g}$ per dose three times a week for two weeks. Animals were euthanized when tumors reached a volume $1500 \mathrm{~mm}^{3}(\mathrm{n}=6)$.

\section{Statistical analysis}

Statistical analysis was performed with SPSS software (19.0; SPSS, Inc., Chicago, IL) as in our previous study and described in supplementary methods [10]. The cutoff criterion for high and low expression of the circRUNX1 was the median value. $P<0.05$ was considered statistically significant. 


\section{Results}

\section{CircRUNX1 was upregulated in LUAD tissues}

Recently, a circular RNA profiling analysis has reported that circRUNX1 (hsa_circ_0002360) was upregulated in human LUAD tissues [18]. However, the biological functions of circRUNX1 on LUAD remains unclear. To further understand the clinical characteristics and significance of the circRUNX1 expression in LUAD, qRT-PCR was used to detect the expression level of circRUNX1 in 110 tumor tissues and paired adjacent non-tumor tissues of LUAD patients. Results indicate that the levels of circRUNX1 was significantly increased in tumor tissue compared with that in paired adjacent normal tissues (Fig. 1a). Next, we analyzed the relationship between circRUNX1 expression and the clinicopathological characteristics of 110 LUAD patients, as listed in Table 1. The results demonstrate that the expression levels of circRUNX1 in TNM stage III was significantly higher than that in TNM stage I-II (Fig. 1b). Additionally, tumor size bigger than $5 \mathrm{~cm}$ had higher circRUNX1 expression than those tumor size smaller than $5 \mathrm{~cm}$ (Fig. 1C). Subsequently, we investigated the prognostic implications of circRUNX1 expression in LUAD patients. Our results demonstrated that patients with higher circRUNX1 expression had a significantly poor prognosis than those with low levels of circRUNX1 (Fig. 1d and e). Multivariate analysis results indicated that circRUNX1 expression is an independent predictor for decreased overall survival (OS) rate and enhanced postoperative relapse rate (Tables 2 and 3). Taken together, our results indicate that circRUNX1 was significantly forced in LUAD tissues and correlated with bad prognosis of LUAD patients. 
Table 1

Correlation between circRUNX1 and clinical characteristics in 110 LUAD patients.

\begin{tabular}{|c|c|c|c|}
\hline \multirow[t]{2}{*}{ Variables } & \multicolumn{2}{|c|}{ circRUNX1 expression level } & \multirow[t]{2}{*}{$P$ value } \\
\hline & Low & High & \\
\hline \multicolumn{4}{|l|}{ Age } \\
\hline $60<$ & 28 & 24 & \multirow[t]{2}{*}{0.567} \\
\hline $60 \geq$ & 27 & 31 & \\
\hline \multicolumn{4}{|l|}{ Gender } \\
\hline Male & 40 & 36 & \multirow[t]{2}{*}{0.536} \\
\hline Female & 15 & 19 & \\
\hline \multicolumn{4}{|l|}{ Smoking status } \\
\hline Smokers & 33 & 43 & \multirow[t]{2}{*}{0.659} \\
\hline Nonsmokers & 22 & 12 & \\
\hline \multicolumn{4}{|l|}{ Tumor stage } \\
\hline$|-| \mid$ & 46 & 30 & \multirow[t]{2}{*}{0.002} \\
\hline III & 9 & 25 & \\
\hline \multicolumn{4}{|c|}{ Lymph node metastasis } \\
\hline Yes & 34 & 29 & \multirow[t]{2}{*}{0.441} \\
\hline No & 21 & 26 & \\
\hline \multicolumn{4}{|l|}{ Tumor size } \\
\hline$\leq 5 \mathrm{~cm}$ & 45 & 18 & \multirow[t]{2}{*}{0.000} \\
\hline$>5 \mathrm{~cm}$ & 10 & 37 & \\
\hline \multicolumn{4}{|l|}{ Differentiation } \\
\hline Well and moderate & 38 & 41 & \multirow[t]{2}{*}{0.672} \\
\hline Poor & 17 & 14 & \\
\hline
\end{tabular}


Table 2

Univariate and Multivariate Analyses of Factors Associated with Overall Survival

OS

Multivariate

Factors

Univariate, $\mathrm{P} \quad \mathrm{HR}$

$\mathrm{HR} \quad 95 \% \mathrm{Cl}$

$P$ value

Sex (Female vs. Male)

0.622

NA

Age (years) $(\leq 60$ vs. $>60)$

0.481

NA

Smoking status (Smokers vs. Nonsmokers)

0.217

NA

Lymph node metastasis (Yes vs. No)

0.035

NS

Differentiation (Well and moderate vs. Poor)

0.042

NS

Tumor size (diameter, $\mathrm{cm})(>5$ vs. $\leq 5)$

0.015

1.375

$1.194-2.492$

0.039

TNM (III vs. I-II)

0.162

NA

CircRUNX1 expression (High vs. Low)

0.004

1.403

$0.837-2.204$

0.027

OS, overall survival; NA, not adopted; NS, not significantly; $95 \% \mathrm{Cl}, 95 \%$ confidence interval; HR, hazard ratio; Cox proportional hazards regression model.

Table 3

Univariate and Multivariate Analyses of Factors Associated with Cumulative Recurrence

\section{Cumulative Recurrence}

Multivariate

Factors

Univariate, $\mathrm{P}$

$\mathrm{HR} \quad 95 \% \mathrm{Cl}$

$P$ value

Sex (Female vs. Male)

0.301

NA

Age (years) ( $\leq 60$ vs. $>60)$

0.582

NA

Smoking status (Smokers vs. Nonsmokers)

0.641

NA

Lymph node metastasis (Yes vs. No)

0.037

NS

Differentiation (Well and moderate vs. Poor)

0.212

NA

Tumor size (diameter, $\mathrm{cm}$ ) (>3 vs. $\leq 3$ )

0.046

NS

TNM (III-IV vs. I-II)

0.032

NS

CircFGFR1 expression (High vs. Low)

0.019

1.627

$1.293-2.705$

0.037

NA, not adopted; NS, not significantly; SCC, Squamous cell carcinoma; $95 \% \mathrm{Cl}, 95 \%$ confidence interval; HR, hazard ratio; Cox proportional hazards regression model. 


\section{Higher level of circRUNX1 expression is correlated with decreased CD8+ $T$ lymphocyte}

Previous studies have been reported that circRNAs dysregulation authorize tumor immune evasion[10, 12], therefore, we wondered whether circRUNX1 overexpression might promote immunosuppressive microenvironment. Then, we explored whether forced circRUNX1 expression exhibited immune evasion signatures by qRT-PCR and IHC analysis in 30 LUAD patient tumor tissues. Interestingly, our results indicated that higher circRUNX1 expression was associated with decreased $C D 8^{+} T$ cells infiltration, while increased Foxp3 ${ }^{+}$cells infiltration (Fig. 2a). Scatter plot analysis demonstrated a negative correlation between circRUNX1 expression and CD8 ${ }^{+} \mathrm{T}$ cells infiltration in the LUAD tissues (Fig. 2b). Moreover, the results from scatter plot analysis also indicated a negative correlation between circRUNX1 expression and Foxp $3^{+} \mathrm{T}$ cells infiltration in the LUAD tissues (Fig. 2c). To determine whether increased circRUNX1 expression induces $\mathrm{CD} 8^{+} \mathrm{T}$ cell dysregulation, $\mathrm{CD} 8^{+} \mathrm{T}$ cells from 30 case LUAD patient tumor tissues were selected. Compared with $\mathrm{CD} 8^{+} \mathrm{T}$ cells in circRUNX1 $1^{\mathrm{Low}}$ tissues, $\mathrm{CD} 8^{+} \mathrm{T}$ cells in circRUNX1 ${ }^{\text {High }}$ tissues demonstrated significant impairment of Granzyme B and perforin secretion when subsequently stimulate with a CD3 antibody (Fig. 2d and e).

\section{Higher level of circRUNX1 expression is correlated with resistance to anti-PD-1 LUAD therapy}

To determine the biological function of circRUNX1 in LUAD sensitivity to anti-PD1 therapy, we then explored retrospective data from 18 recurrent LUAD patients receiving anti-PD-1 therapy who had undergone primary tumor resection 2-24 months before the immunotherapy. Then, circRUNX1 expression in LUAD tissues were measured using qRT-PCR (Fig. 3a), and Kaplan-Meier survival analysis demonstrated that the progression-free survival for the circRUNX1-high group was much shorter than that for the circRUNX1-low group (Fig. 3b). To further determine the effects of circRUNX1 expression on the sensitivity of LUAD to anti-PD-1 immunotherapy, we analyzed circRUNX1 expression in five human LUAD cell lines using qRT-PCR (Supplementary Fig. 1a). To verify the biological functions of circRUNX1 in LUAD cells, we designed an overexpression lentivirus (circRUNX1-OE) transfected them into the NCI-H1975 and Clau-3 cell lines to upregulate circRUNX1 expression. The qRT-PCR results indicated that transfection of overexpressing circRUNX1 lentivirus vector resulted in significant forced circRUNX1 expression levels, while the expression levels of RUNX1 mRNA is not changed (Supplementary Fig. 1b and c). In addition, we also designed two shRNAs lentivirus vector targeting the back splicing site of circRUNX1 and transfected them into the PC-9 and HCC-827 cell lines to knockdown circRUNX1 (Supplementary Fig. 1d and e). Then, we explored the anti-tumor effects of the PD-1 antibody in humanized NSG mice that received LUAD cells with forced circRUNX1 expression or the respective mock cells ( $\mathrm{NCl}-\mathrm{H} 1975-\mathrm{Mock}, \mathrm{NCl}-$ $\mathrm{H} 1975$-circRUNX1). Compared to that of the NCl-H1975-Mock cell group, the tumor growth in the NClH1975-circRUNX1 cell recipient xenograft mice showed a significantly phenotype of resistance to anti-PD1 immunotherapy, and the xenograft mice had a decreased survival time (Fig. 3c-e). To further explore the molecular mechanism of circRUNX1-mediated LUAD resistance to anti-PD-1 immunotherapy, we have examined the effect of circRUNX1 on the PD-L1 expression on LUAD cells. Our results showed that the 
expression of PD-L1 was not changed significantly when circRUNX1 was overexpressed or knockdown in the LUAD cell lines (Fig. $3 f$ and $g$ ). Above results indicate that circRUNX1-mediated LUAD resistance to anti-PD-1 immunotherapy in a PD-1/PD-L1-independent manner.

\section{CircRUNX1 acts as a sponge for miR-4739 in LUAD cells}

To reveal the molecular mechanisms of circRUNX1-promoting LUAD immune evasion, we predicted the potential interaction between circRUNX1 and miRNA using the Star Base v3.0. We found that circRUNX1 can bind to 9 potential miRNAs based on StarBase v3.0. To determine the critical miRNA sponging circRUNX1, we performed a pulldown assay with a biotinylated circRUNX1 probe. As shown in Fig. 4a, we selected miR-4739 was the significantly captured miRNA for circRUNX1. To determine whether circRUNX1 and miR-4739 are co-localized, the FISH assay was used to NCl-H1975 and HCC-827 cells, and the results showed that circRUNX1 and miR-4739 were co-located major in the cytoplasm (Fig. 4b). AGO2 antibodymediated RIP assay showed that circRUNX1 and miR-4739, but not circANRIL (a circRNA verified to not bind to AGO2 protein [19]), were significantly captured in NCl-H1975 and HCC-827 cells (Fig. 4c). To further verify the predicted interaction between circRUNX1 and miR-4739, we performed RNA-pull down assays with a biotinylated miR-4739 mimics. In both NCl-H1975 and HCC-827 cells, circRUNX1 was significantly captured (Fig. 4d). In addition, we performed a luciferase assay using miR-4739 mimics or negative control (NC) mimics co-transfected with pLG3 luciferase reporter plasmids (which contained a wild-type or miR-4739-target mutant circRUNX1 sequence) into HEK-293T cells. Compared with the NC mimics, miR-4739 inhibited the luciferase reporter activity significantly in the cells with the wild-type circRUNX1 sequence but not the cells with either the wild-type- or the miR-4739-target mutant circRUNX1 sequence (Fig. 4e and f). Importantly, the levels of circRUNX1 did not show obvious changes after miR4739 expression was either upregulated or knocked down, and the levels of miR-4739 did not show obvious changes after circRUNX1 expression was either was overexpressed or knocked down (Fig. 4gj;Supplementary Fig. 2a and b). These results demonstrate that miR-4739 and circRUNX1 are likely not degraded by each other.

\section{PCSK9 was a direct target of miR-4739}

To further reveal the underlying biological mechanisms of miR-4739 and its down-stream critical molecular, then, Targetscan was used to predict the potential target mRNAs of miR-4739. Interestingly, PCSK9, an immune-related gene is a potential target mRNA of miR-4739 (Fig. 5a). Dual-luciferase reporter assay results demonstrated that the relative luciferase activity of PCSK9 was obviously inhibited, suggesting that the PCSK9 mRNA is a target of miR-4739 (Fig. 5b). Then, the expression of PCSK9 mRNA and protein was detected in miR-4739 overexpression LUAD cell lines. The results indicated that the expression of PCSK9 mRNA and protein was significantly decreased in miR-4739-overexpressing cell lines (Fig. $5 \mathrm{c}$ and d). Next, the expression of PCSK9 mRNA and protein was examined in circRUNX1 overexpression and knockdown LUAD cell lines, respectively. Our results showed that the expression of PCSK9 mRNA and protein was significantly increased in circRUNX1-overexpressing cell lines, while decreased in circRUNX1 knockdown cell lines (Fig. 5e-h). Next, a miR-4739 target sequence mutant circRUNX1 overexpression lentivirus (circRUNX1-mOE) transfected into the NCI-H1975 and Clau-3 cell lines to further determined that circRUNX1-mediated PCSK9 upregulation was in a miR-4739-depedent 
manner (Supplementary Fig. 3a and b). Our results showed that the expression of PCSK9 mRNA and protein was not significantly increased in mutant circRUNX1-overexpressing cell lines (Fig. $5 \mathrm{i}$ and j).

\section{Knocking out PCSK9 reverses circRUNX1-induced LUAD resistance to anti-PD-1 immunotherapy}

Recently, it has been reported that higher levels of PCSK9 downregulates the expression of MHC I proteins on cancer cells and induces tumor resistance to anti-PD1 immunotherapy [15]. Therefore, we conjectured that circRUNX1 promotes LUAD resistance to anti-PD-1 immunotherapy via the miR-4739/PCSK9 axis. To further determine whether circRUNX1-induced resistance to anti-PD-1 immunotherapy of LUAD through the miR-4739/PCSK9 axis, we established PCSK9-knockout NCl-H1975 cells (NCl-H1975-KOP) (Fig. 6a). The PCSK9 protein did not detected after circRUNX1 expression was upregulated in the $\mathrm{NCl}-\mathrm{H} 1975$ cells (Fig. 6b). Furthermore, we analyzed the anti-tumor effects of the PD-1 antibody in humanized NSG mice that received $\mathrm{NCl}-\mathrm{H} 1975-\mathrm{KOP}$ cells with forced circRUNX1 expression or the respective mock cells ( $\mathrm{NCl}-$ H1975-KOP-Mock, NCl-H1975-KOP-circRUNX1). Compared to that of the NCl-H1975-KOP-Mock cell group, the tumor growth in the NCl-H1975-KOP-circRUNX1 cell recipient xenograft mice showed no obviously phenotype of resistance to anti-PD1 immunotherapy (Fig. 6c-e). In addition, our results showed that the levels of MHC I was significantly decreased when circRUNX1 was overexpressed in the LUAD cells (Fig. 6f). To further explore the relationship between the circRUNX1 and immune evasion, we measured the $\mathrm{MHC}$ I expression in tissues in the above 30 cases of LUAD tissues (Fig. $6 \mathrm{~g}$ ). The results from a scatter plot analysis showed a negative correlation between circRUNX1 expression and MHC I expression in the LUAD tissues (Fig. 6h). These results indicate that circRUNX1 may exert its immunosuppressive effects by circRUNX1/ miR-4739/PCSK9/MHC I axis.

\section{Discussion}

PD-1 monoclonal antibody-induced anti-tumor effect relies on restoration of the cancer-immunity system, initiated by immune effector cells including cytotoxic T lymphocytes, natural killer cells, et al [20, 21]. Importantly, efficacious MHC I-mediated presentation of tumor-specific antigens one of the critical factors for anti-PD-1-based immunotherapy curative effect [22]. Anti-PD-1-based immunotherapy have verified to be a critical therapeutic approach for LUAD. Mechanistically, anti-PD-1 immunotherapy acts by breaking the interaction between PD-1 and PD-L1, which are responsible for the promotion of cancer immune evasion [23]. However, the application of anti-PD-1 immunotherapy in LUAD is limited by the primary or acquired drug resistance $[23,24]$. Recently, circRNAs have been reported to play vital roles in the tumorigenesis and progression of tumors $[25,26]$. Previous studies have identified several irregular expressed circRNAs in LUAD that are dysregulated in LUAD tissues and function as tumor suppressor genes or oncogenes. For example, circRNA-ENO1 can promote glycolysis and tumor progression by binding to miR-22-3p and upregulating the expression of ENO1 in LUAD [27]. CircDCUN1D4 can upregulate thioredoxin-interacting protein (TXNIP) expression in LUAD by facilitating the interaction between HuR protein and TXNIP mRNA, thus inhibiting cell metastasis and glycolysis [28]. Wang et al. found that forced circRNA-002178 expression promotes T-cell exhaustion via regulation of the miR- 
34/PD-L1 signaling pathway [29]. Here, our results demonstrate that circRUNX1 is a critical regulator of immune evasion in LUAD cells.

Recent studies have confirmed that circRNAs are participate in the progression and immune evasion of various cancers via acting as miRNA sponges, therefore, modulating protein translation $[11,25]$. In this study, we used StarBase v3.0 to predict the potential miRNAs interaction with circRUNX1, then confirmed by biotin-coupled probe pull-down, AGO2-mediated RIP, and dual luciferase reporter assays. Our results showed that circRUNX1 was able to bind with StarBase v3.0. Furthermore, FISH assay confirmed the colocalization between circRUNX1 and miR-4739 in LUAD cells. We next attempted to explore the biological role and molecular mechanism of miR-4739 in LUAD cells and revealed that miR-4739 executed functions in LUAD immune evasion by targeting PCSK9. To further verify whether the biological function of h circRUNX1 in LUAD cells was associated with miR-4739/PCSK9 pathway, we carried out a block experiment using CRISPR-Cas 9 to knock out PCSK9 expression. Our results demonstrated that circRUNX1 promotes LUAD immune evasion by upregulating PCSK9 expression via sponging miR-4739.

Decreased MHC I molecular expression is an important mechanism of cancer immune evasion [8]. Importantly, deleted MHC I expression induced cancer resistance to PD-1/PD-L1-based immunotherapy [30]. Inhibiting PCSK9 expression or blocking PCSK9 by antibody increases the expression of MHC I proteins on the cancer cell surface, thus, promoting robust intratumoral infiltration of cytotoxic $T$ cells [15]. Interestingly, we found that PCSK9 protein levels were significantly increased in circRUNX1 overexpression LUAD cells. Here, our data reported the critical role of circRUNX1 in tumor resistance to anti-PD-1 immunotherapy and inhibition of MHC I signaling pathway in LUAD immune evasion.

\section{Conclusions}

Taken together, our study indicates that circRUNX1 is overexpressed in LUAD and its upregulation is correlated with poor prognosis. Mechanism, circRUNX1 promotes LUAD immune evasion by acting as a sponge for miR-4739, which decreases the activity of PCSK9/MHC I axis. These results demonstrate that circRUNX1 is a promising novel biomarker and therapeutic target in LUAD.

\section{Abbreviations}

LUAD: Lung adenocarcinoma; PD-1: Programmed death-1; PD-L1: Programmed death ligand 1; circRNAs: circular RNA; PCSK9: Proprotein convertase subtilisin/kexin type 9; qRT-PCR: Quantitative real-time polymerase chain reaction; IHC: Immunohistochemistry; NSCLC: Non-small cell lung cancer; MHC I: Histocompatibility complex I; CXCR4: C-X-C motif chemokine receptor 4; circRIP: CircRNA precipitation; RIP: RNA immunoprecipitation; FISH: Fluorescence in situ hybridization.

\section{Declarations}

- Ethics approval and consent to participate 
Ethical approval was obtained from the Zhongshan Hospital Research Ethics Committee.

- Consent for publication

Not applicable.

- Availability of data and materials

All data generated or analyzed in this study are included in this article and its supplementary information files.

- Competing interests

The authors declare no conflicts of interest.

- Funding

This study was funded by the National Natural Science Foundation of China (82072575).

\section{- Authors' contributions}

PF Zhang and Y Fan conceived and designed the experiments; PF Zhang, YF Xu, and LX Zhang performed the experiments; PF Zhang and J Wu analyzed the data; PF Zhang and Y Fan wrote the paper. All authors read and approved the final manuscript.

\section{- Acknowledgements}

Not applicable.

\section{References}

1. Manjili MH, Payne KK: Cancer immunotherapy: Re-programming cells of the innate and adaptive immune systems. Oncoimmunology 2012, 1(2):201-204.

2. Doroshow DB, Sanmamed MF, Hastings K, Politi K, Rimm DL, Chen L, Melero I, Schalper KA, Herbst RS: Immunotherapy in Non-Small Cell Lung Cancer: Facts and Hopes. Clin Cancer Res 2019, 25(15):4592-4602.

3. Tseng D, Padda SK, Wakelee HA: Perspectives on Acquired Resistance to PD-1 Axis Inhibitors in Patients with Non-Small Cell Lung Cancer. J Thorac Oncol 2018, 13(6):741-744.

4. Gettinger SN, Wurtz A, Goldberg SB, Rimm D, Schalper K, Kaech S, Kavathas P, Chiang A, Lilenbaum $\mathrm{R}$, Zelterman $\mathrm{D}$ et al: Clinical Features and Management of Acquired Resistance to PD-1 Axis Inhibitors in 26 Patients With Advanced Non-Small Cell Lung Cancer. J Thorac Oncol 2018, 13(6):831-839.

5. Siegel RL, Miller KD, Jemal A: Cancer statistics, 2019. CA Cancer J Clin 2019, 69(1):7-34. 
6. Burr ML, Sparbier CE, Chan KL, Chan YC, Kersbergen A, Lam EYN, Azidis-Yates E, Vassiliadis D, Bell CC, Gilan $O$ et al: An Evolutionarily Conserved Function of Polycomb Silences the MHC Class I Antigen Presentation Pathway and Enables Immune Evasion in Cancer. Cancer Cell 2019, 36(4):385401 e388.

7. Le Maux Chansac B, Moretta A, Vergnon I, Opolon P, Lecluse Y, Grunenwald D, Kubin M, Soria JC, Chouaib S, Mami-Chouaib F: NK cells infiltrating a MHC class I-deficient lung adenocarcinoma display impaired cytotoxic activity toward autologous tumor cells associated with altered NK celltriggering receptors. J Immunol 2005, 175(9):5790-5798.

8. Shukla A, Cloutier M, Appiya Santharam M, Ramanathan S, Ilangumaran S: The MHC Class-I Transactivator NLRC5: Implications to Cancer Immunology and Potential Applications to Cancer Immunotherapy. Int J Mol Sci 2021, 22(4).

9. Gu SS, Zhang W, Wang X, Jiang P, Traugh N, Li Z, Meyer C, Stewig B, Xie Y, Bu X et al: Therapeutically increasing MHC-I expression potentiates immune checkpoint blockade. Cancer Discov 2021.

10. Zhang PF, Pei X, Li KS, Jin LN, Wang F, Wu J, Zhang XM: Circular RNA circFGFR1 promotes progression and anti-PD-1 resistance by sponging miR-381-3p in non-small cell lung cancer cells. Mol Cancer 2019, 18(1):179.

11. Zhou WY, Cai ZR, Liu J, Wang DS, Ju HQ, Xu RH: Circular RNA: metabolism, functions and interactions with proteins. Mol Cancer 2020, 19(1):172.

12. Zhang PF, Gao C, Huang XY, Lu JC, Guo XJ, Shi GM, Cai JB, Ke AW: Cancer cell-derived exosomal circUHRF1 induces natural killer cell exhaustion and may cause resistance to anti-PD1 therapy in hepatocellular carcinoma. Mol Cancer 2020, 19(1):110.

13. Wang S, Zhang K, Tan S, Xin J, Yuan Q, Xu H, Xu X, Liang Q, Christiani DC, Wang M et al: Circular RNAs in body fluids as cancer biomarkers: the new frontier of liquid biopsies. Mol Cancer 2021, 20(1):13.

14. Bhattacharya A, Chowdhury A, Chaudhury K, Shukla PC: Proprotein convertase subtilisin/kexin type 9 (PCSK9): A potential multifaceted player in cancer. Biochim Biophys Acta Rev Cancer 2021, 1876(1):188581.

15. Liu X, Bao X, Hu M, Chang H, Jiao M, Cheng J, Xie L, Huang Q, Li F, Li CY: Inhibition of PCSK9 potentiates immune checkpoint therapy for cancer. Nature 2020, 588(7839):693-698.

16. Zhang PF, Wei CY, Huang XY, Peng R, Yang X, Lu JC, Zhang C, Gao C, Cai JB, Gao PT et al: Circular RNA circTRIM33-12 acts as the sponge of MicroRNA-191 to suppress hepatocellular carcinoma progression. Mol Cancer 2019, 18(1):105.

17. Choi B, Lee JS, Kim SJ, Hong D, Park JB, Lee KY: Anti-tumor effects of anti-PD-1 antibody, pembrolizumab, in humanized NSG PDX mice xenografted with dedifferentiated liposarcoma. Cancer Lett 2020, 478:56-69.

18. Wang C, Tan S, Liu WR, Lei Q, Qiao W, Wu Y, Liu X, Cheng W, Wei YQ, Peng Y et al: RNA-Seq profiling of circular RNA in human lung adenocarcinoma and squamous cell carcinoma. Mol Cancer 2019, 18(1):134. 
19. Holdt LM, Stahringer A, Sass K, Pichler G, Kulak NA, Wilfert W, Kohlmaier A, Herbst A, Northoff BH, Nicolaou $A$ et al: Circular non-coding RNA ANRIL modulates ribosomal RNA maturation and atherosclerosis in humans. Nat Commun 2016, 7:12429.

20. Chen DS, Mellman I: Elements of cancer immunity and the cancer-immune set point. Nature 2017, 541(7637):321-330.

21. Cichocki F, Bjordahl R, Gaidarova S, Mahmood S, Abujarour R, Wang H, Tuininga K, Felices M, Davis $\mathrm{ZB}$, Bendzick $\mathrm{L}$ et al: iPSC-derived NK cells maintain high cytotoxicity and enhance in vivo tumor control in concert with T cells and anti-PD-1 therapy. Sci Trans/ Med 2020, 12(568).

22. Chowell D, Krishna C, Pierini F, Makarov V, Rizvi NA, Kuo F, Morris LGT, Riaz N, Lenz TL, Chan TA: Evolutionary divergence of HLA class I genotype impacts efficacy of cancer immunotherapy. Nat Med 2019, 25(11):1715-1720.

23. Qu J, Mei Q, Liu L, Cheng T, Wang P, Chen L, Zhou J: The progress and challenge of anti-PD-1/PD-L1 immunotherapy in treating non-small cell lung cancer. Ther Adv Med Oncol 2021, 13:1758835921992968.

24. Boyero L, Sanchez-Gastaldo A, Alonso M, Noguera-Ucles JF, Molina-Pinelo S, Bernabe-Caro R: Primary and Acquired Resistance to Immunotherapy in Lung Cancer: Unveiling the Mechanisms Underlying of Immune Checkpoint Blockade Therapy. Cancers (Basel) 2020, 12(12).

25. Yang X, Ye T, Liu H, Lv P, Duan C, Wu X, Jiang K, Lu H, Xia D, Peng E et al: Expression profiles, biological functions and clinical significance of circRNAs in bladder cancer. Mol Cancer 2021, 20(1):4.

26. Long F, Lin Z, Li L, Ma M, Lu Z, Jing L, Li X, Lin C: Comprehensive landscape and future perspectives of circular RNAs in colorectal cancer. Mol Cancer 2021, 20(1):26.

27. Zhou J, Zhang S, Chen Z, He Z, Xu Y, Li Z: CircRNA-ENO1 promoted glycolysis and tumor progression in lung adenocarcinoma through upregulating its host gene ENO1. Cell Death Dis 2019, 10(12):885.

28. Liang $Y$, Wang $H$, Chen B, Mao Q, Xia W, Zhang T, Song X, Zhang Z, Xu L, Dong G et al: circDCUN1D4 suppresses tumor metastasis and glycolysis in lung adenocarcinoma by stabilizing TXNIP expression. Mol Ther Nucleic Acids 2021, 23:355-368.

29. Wang J, Zhao X, Wang Y, Ren F, Sun D, Yan Y, Kong X, Bu J, Liu M, Xu S: circRNA-002178 act as a ceRNA to promote PDL1/PD1 expression in lung adenocarcinoma. Cell Death Dis 2020, 11(1):32.

30. Dusenbery AC, Maniaci JL, Hillerson ND, Dill EA, Bullock TN, Mills AM: MHC Class I Loss in Triplenegative Breast Cancer. A Potential Barrier to PD-1/PD-L1 Checkpoint Inhibitors. Am J Surg Pathol 2021.

\section{Figures}


$\mathrm{a}$

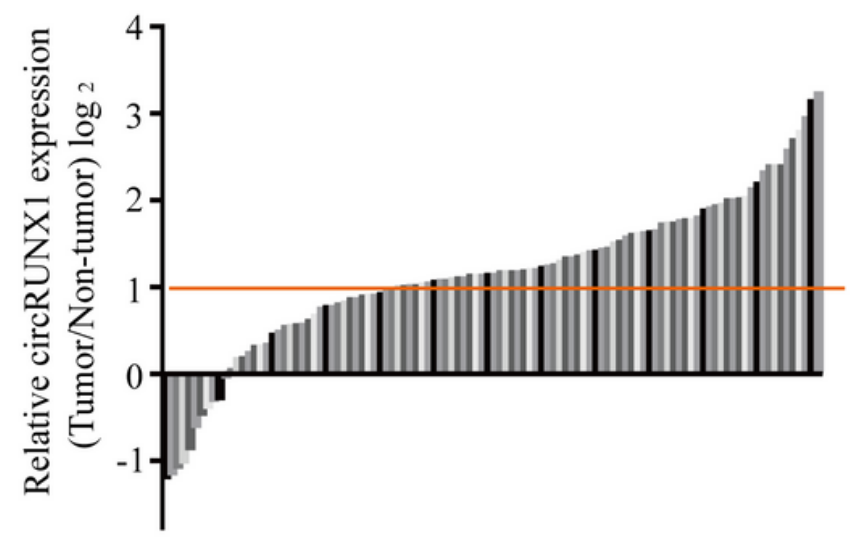

d

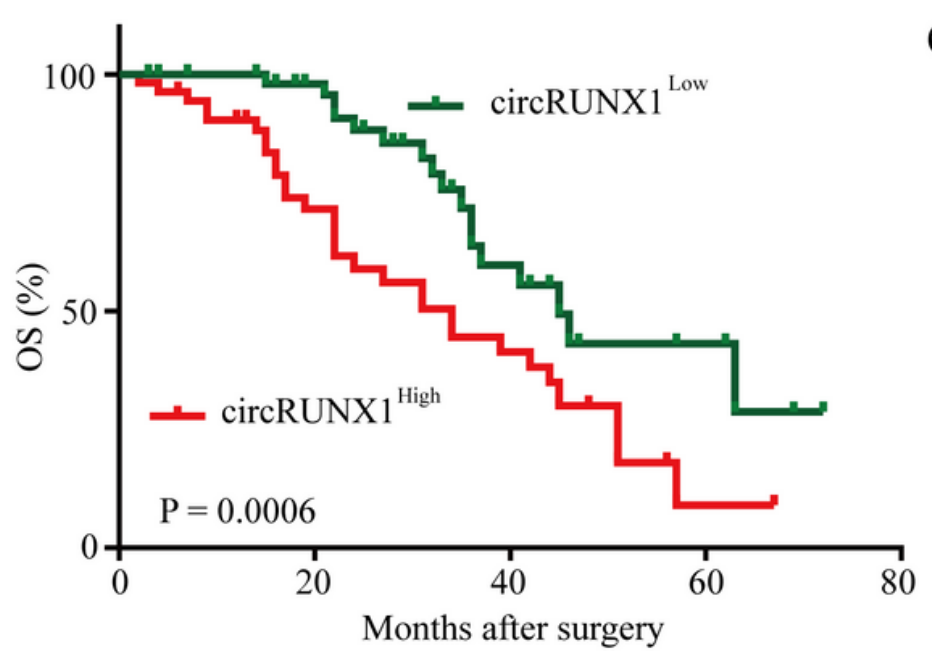

$\mathrm{b}$

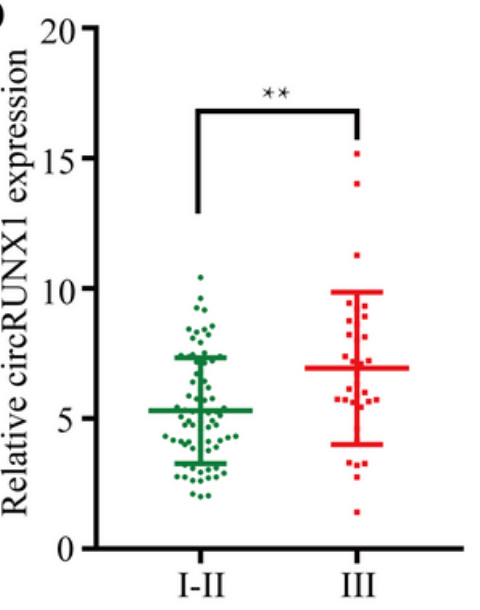

$\mathrm{C}$

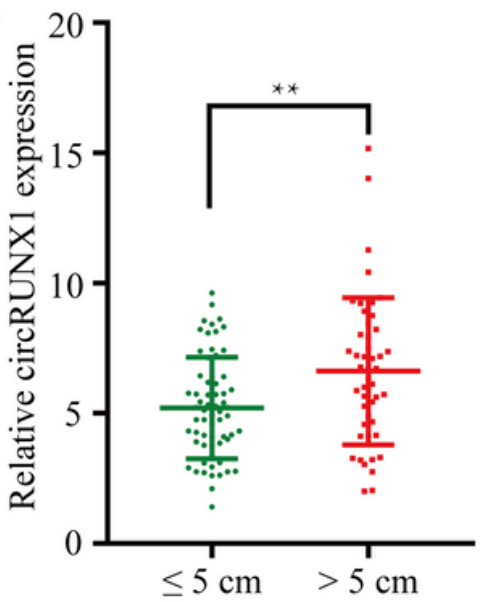

e

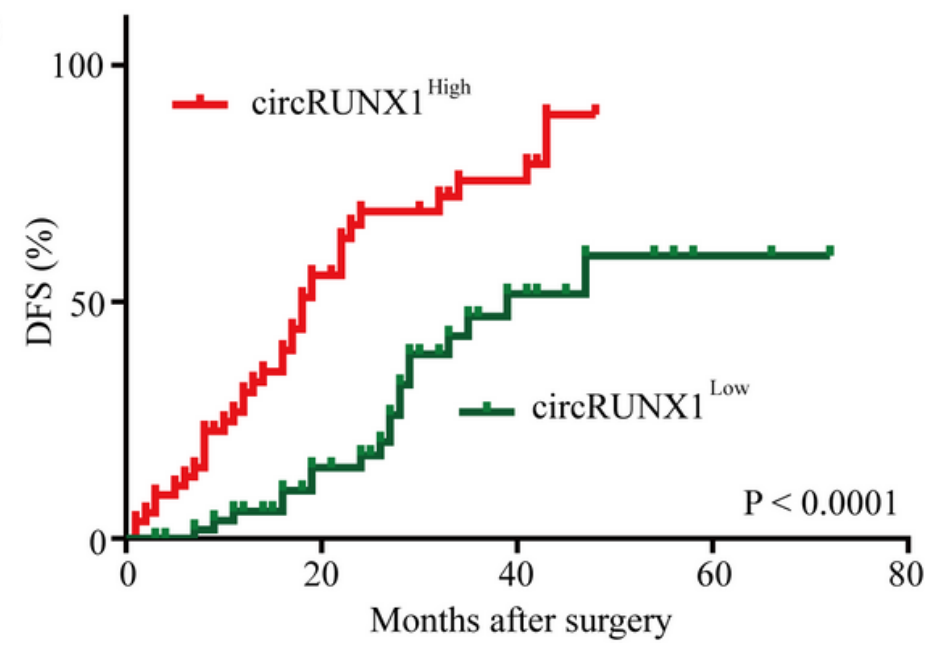

Figure 1

CircRUNX1 is upregulated in LUAD tissues. a The expression of circRUNX1 levels in 110 pairs of LUAD and matched adjacent nontumor tissues was detected by qRT-PCR. The results were shown as log2 (T/N). b A total of 110 patients were divided into I-II stage and III stage groups. The diagram shows circRUNX1 expression in each group. c A total of 110 patients were divided into $\leq 5 \mathrm{~cm}$ and $>5 \mathrm{~cm}$ size groups. The diagram shows circRUNX1 expression in each group. ( $\mathrm{d}$ and $\mathrm{e}$ ) Kaplan-Meier analysis of overall survival and disease-free survival in 110 patients with LUAD according to circRUNX1 expression (log-rank test). Data are presented as the means \pm SD of three independent experiments. ${ }^{\star *} \mathrm{P}<0.01$. 

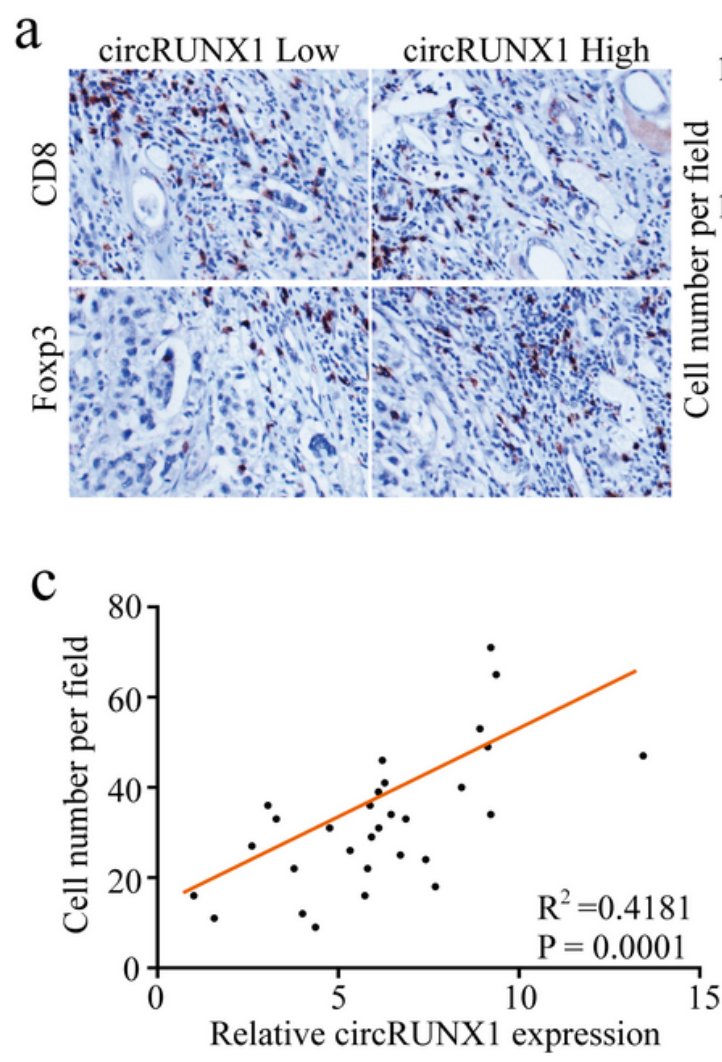

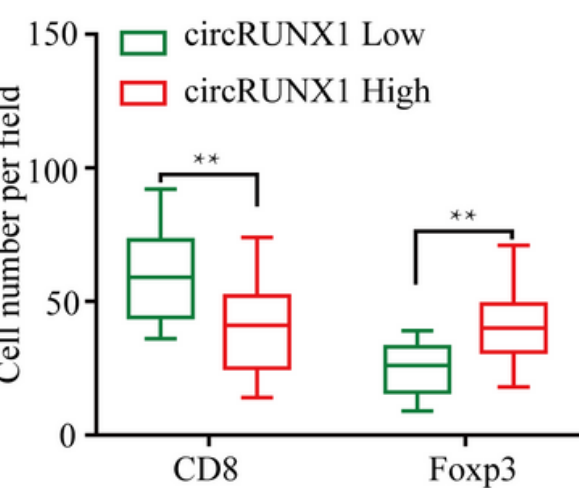

d

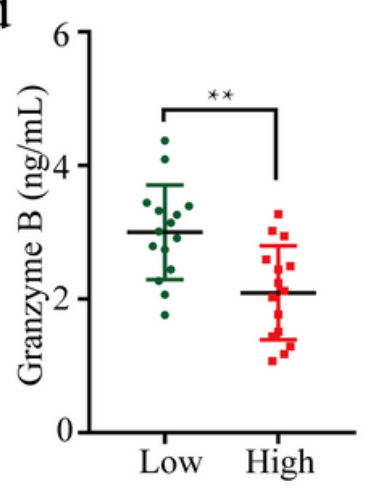

b

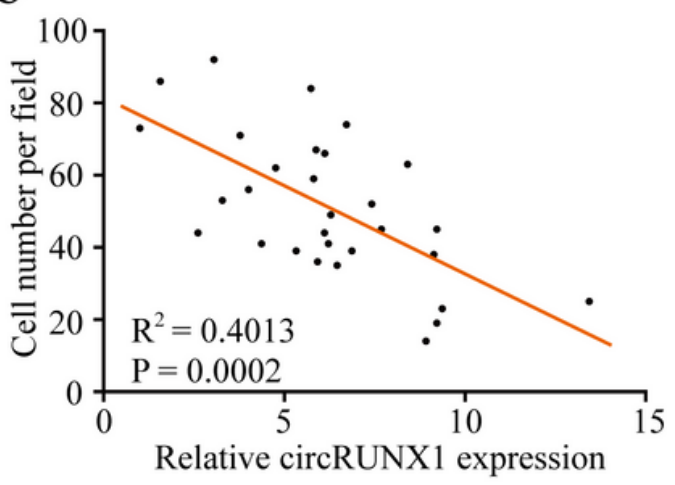

$\mathrm{e}$

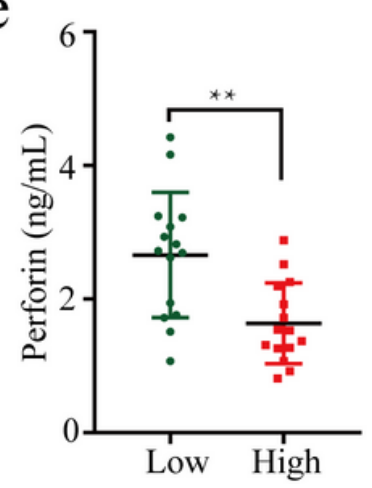

Figure 2

CircRUNX1 related to immune evasion in LUAD tissues. a CD8 or Foxp3-positive cells in the LUAD tissues were analyzed by IHC. b A negative correlation between circRUNX1 and CD8-positive cells was observed in tumor tissues from LUAD patients $(R 2=0.4013 ; P=0.0002)$. c A positive correlation between circRUNX1 and Foxp3-positive cells was observed in in tumor tissues from LUAD patients $(R 2=0.4181 ; P$ $=0.0001)$. $d$ and e A total of 30 LUAD tissues were divided into circRUNX1 High and circRUNX1Low groups. The diagram shows Granzyme B and perforin secretion of CD8-positive cells in each group. $* * \mathrm{P}<$ 0.01 . 

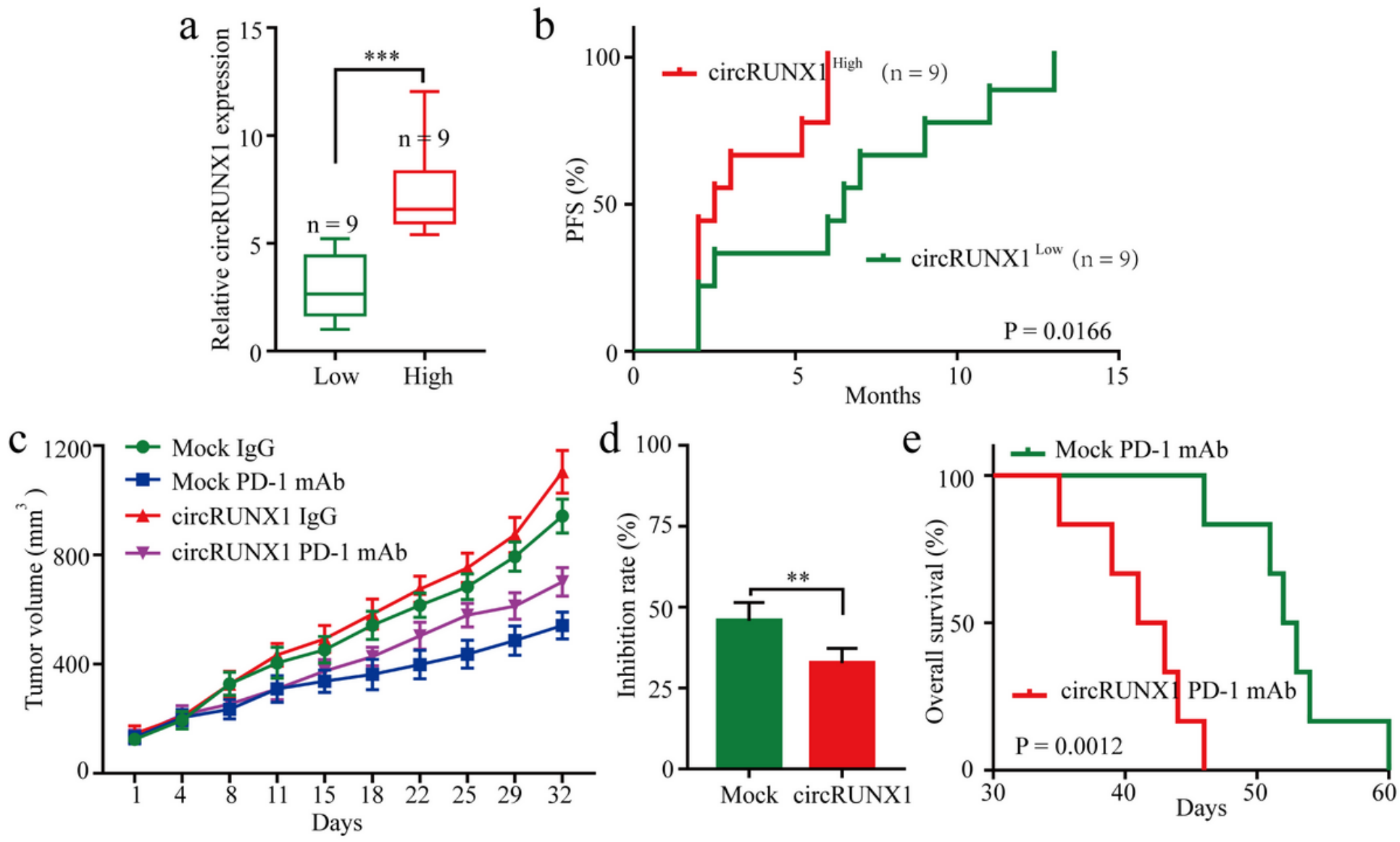

\section{Figure 3}

Forced circRUNX1 expression correlate with resistance to anti-PD1 immunotherapy in LUAD. a The expression of circRUNX1 levels in 18 tumor tissues of LUAD patient. b Kaplan-Meier analysis of PFS in 18 patients with LUAD according to circRUNX1 expression (log-rank test). c NCl-H1975-Mock or NCl-H1975circRUNX1 cells were subcutaneously injected into huNSG mice, when tumors volume reached about 100 mm3 the mice were treated with IgG or PD-1 antibody (The data are presented as the mean $\pm S D ; n=6$ ). $d$ The data are expressed as the inhibition rate of tumors. e Comparison of the OS curves for mice with forced circRUNX1 expression or Mock control in xenograft LUAD tumors that were treated with PD-1 antibody. ${ }^{\star *} \mathrm{P}<0.01,{ }^{\star * \star} \mathrm{P}<0.001$. 
a

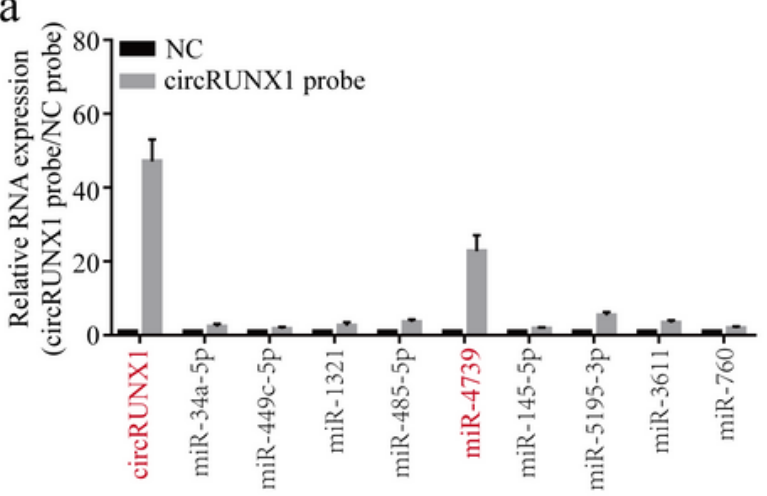

C

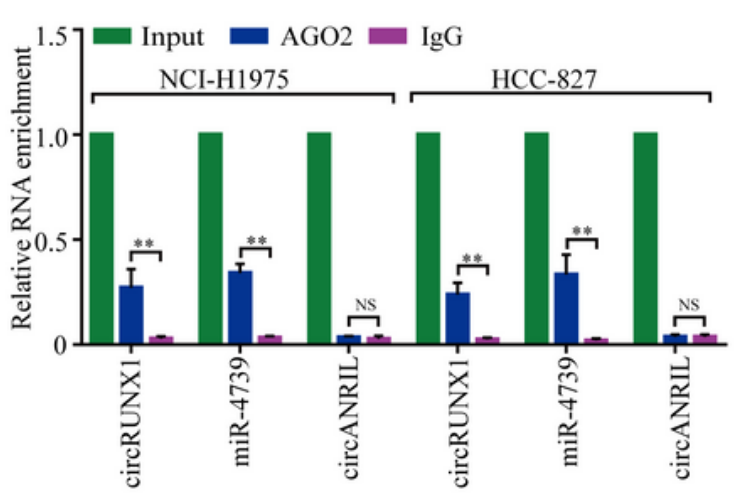

e

wt circRUNX1 5 ' cacGCCCAACC - CUCGUGCCUCCCUg 3'

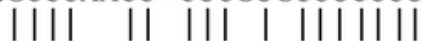
miR-4739 3' uccCGGGGAGGCGAGGA- GGAGGGAa 5' III |||||||||||| $\mid$ mu circRUNX1 5' cacGCCCAACC - CUCGUGGGAGGGAg 3'

$\mathrm{f}$ b
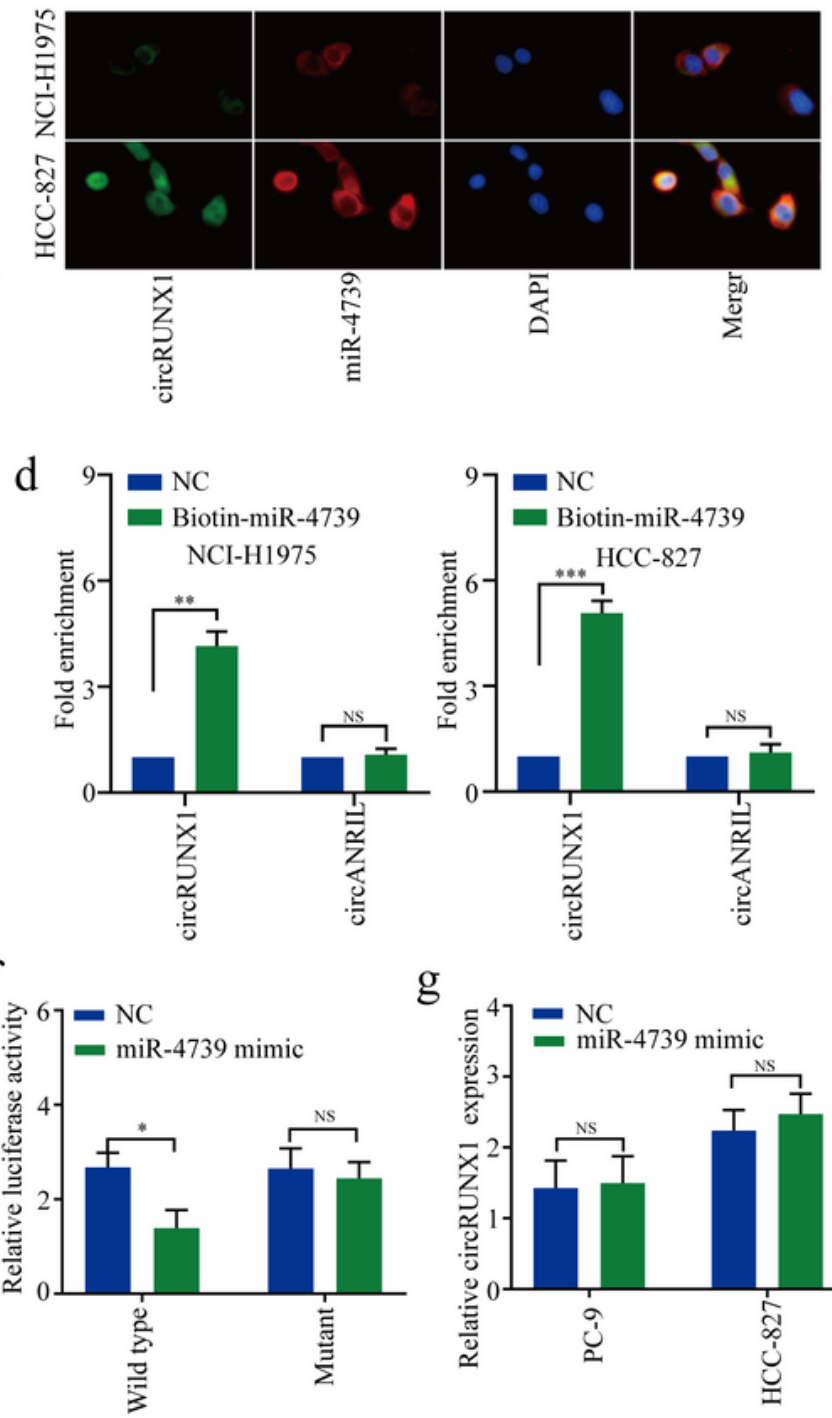

$\mathrm{g}$

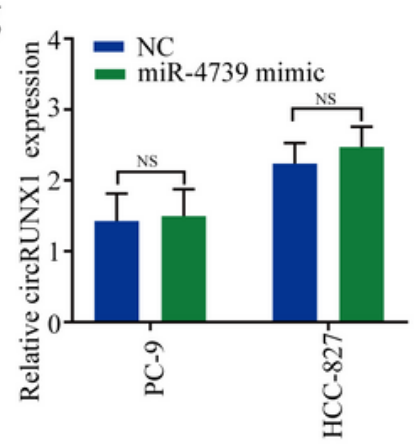

$\mathrm{h}$

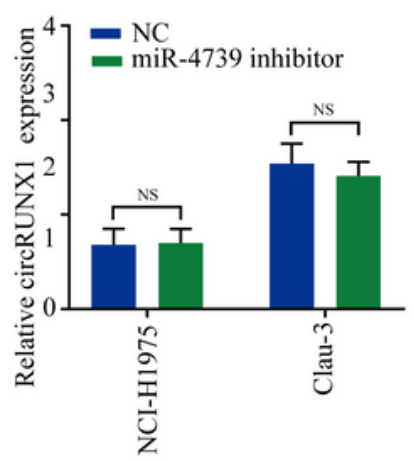

i

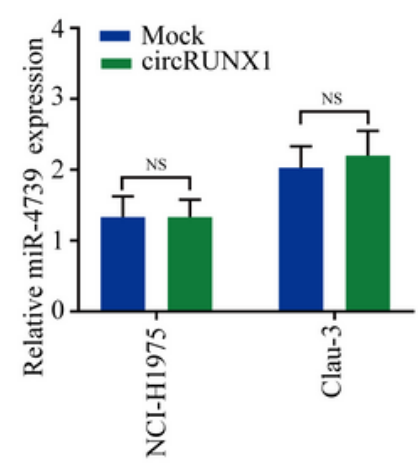

$\mathrm{j}$

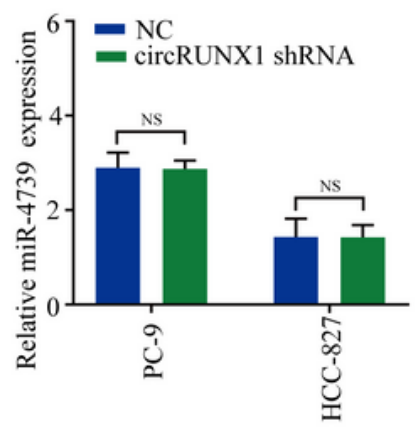

Figure 4

CircRUNX1 acts as a sponge for miR-4739. a CircRIP analysis was performed to explore the miRNAs bound to circRUNX1 in HCC-827 cells. b The FISH results demonstrated that both of circRUNX1 and miR4739 are localized in the cytoplasm. c RIP experiments were performed using an AGO2 antibody with NClH1975 and HGC-827 cell extracts. d The level of circRUNX1 in the streptavidin-captured fractions of NCIH1975 and HGC-827 cell lysates after transfection with biotinylated miR-4739 or the negative control. e 
Putative binding sites of miR-4739 in circRUNX1 were predicated via StarBase v3.0. $f$ The luciferase activity of pLG3-circRUNX1 or mutant pLG3-circRUNX1 in the HEK-293 T cells after cotransfection with miR-4739 mimic or negative control. g The relative level of circRUNX1 was detected by qRT-PCR in PC-9 and HCC-827 cell lines transfected with miR-4739 mimic or negative control. $h$ The relative level of circRUNX1 was detected by qRT-PCR in NCl-H1975 and Clau-3 cell lines transfected with miR-4739 siRNA or negative control. i The relative level of miR-4739 was detected by qRT-PCR in NCl-H1975 and Clau-3 cell lines transfected with circRUNX1 or mock control. i The relative level of miR-4739 was detected by qRT-PCR in PC-9 and HCC-827 cell lines transfected with circRUNX1 shRNA or negative control. Data are presented as the means \pm SD of three independent experiments. ${ }^{\star} P<0.05,{ }^{\star} \mathrm{P}<0.01,{ }^{\star} * \star \mathrm{P}<0.001$. NS, no significant.

a

wt PCSK9 5' ggugccaggaaGCUC - - CCU CCCUc 3'

| || || || ||||

miR-4739 3' ucceggggaggCGAGGAGGAGGGAa 5'

| || || || ||

mu PCSK9 5' ggugccaggaaGCUC - - GGAGGGAc 3' b

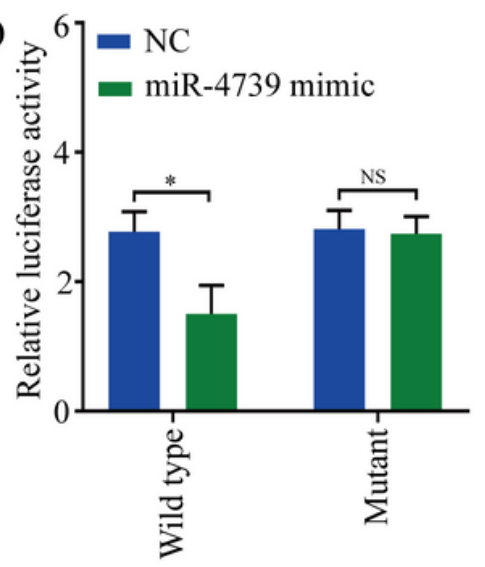

f

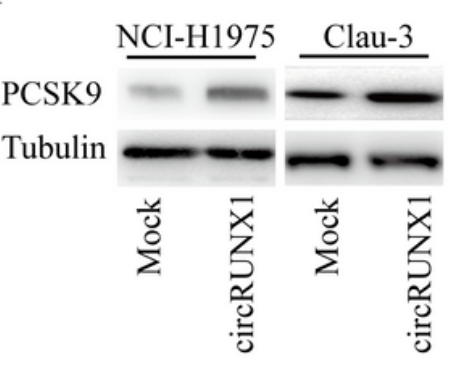

d
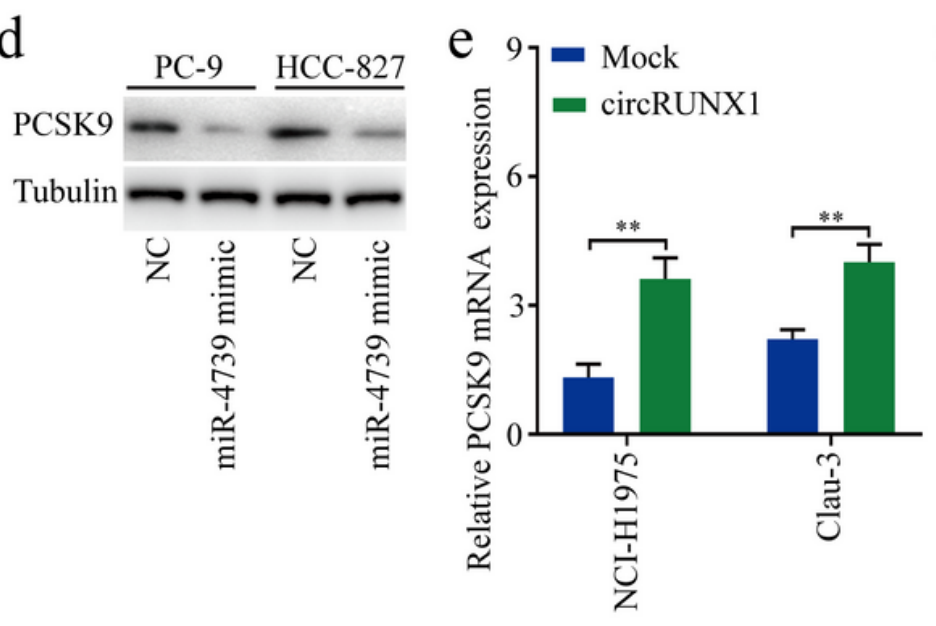

$\mathrm{h}$

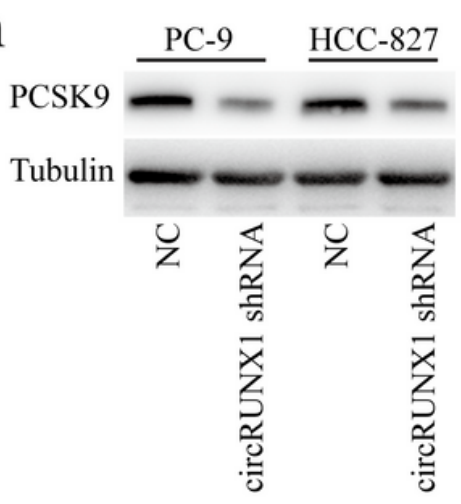

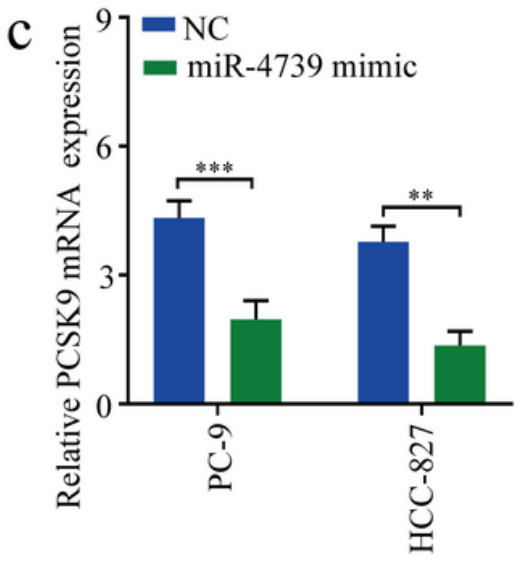

g $97=\mathrm{NC}$

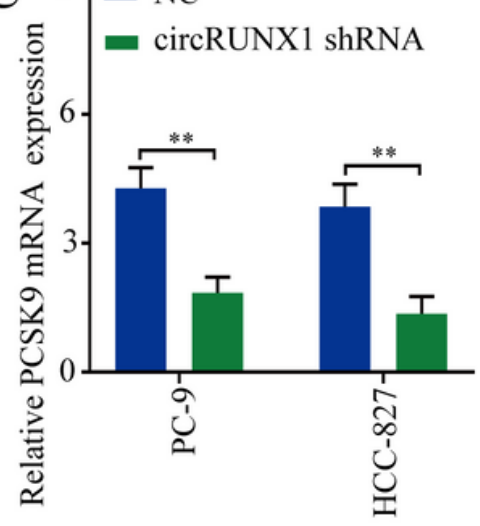

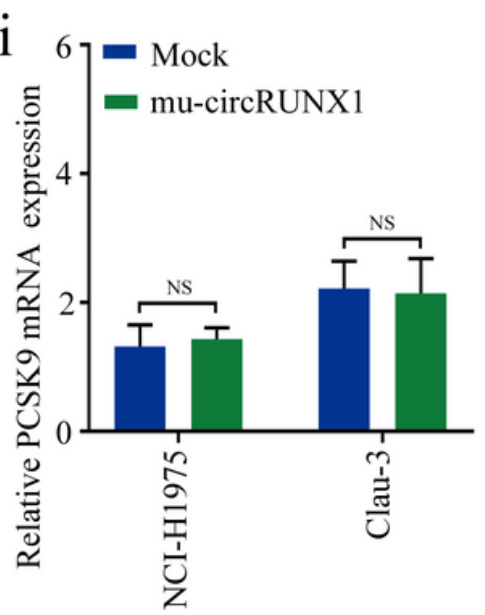

j

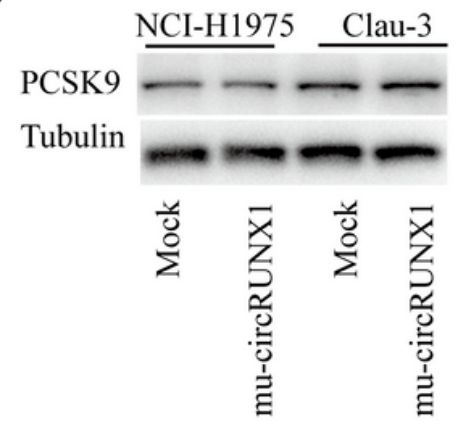




\section{Figure 5}

CircRUNX1 may upregulate PCSK9 expression via sponge miR-4739 in LUAD cells. a The interaction of miR-4739 and PCSK9 mRNA was predicted by StarBase v3.0. b The luciferase activity of pLG3-PCSK9 or mutant pLG3-PCSK9 in the HEK-293 T cells after cotransfection with miR-4739 mimic or negative control. $c$ and $d$ The mRNA and protein levels of PCSK9 was examined by qRT-PCR or western blot in the PC-9 and HCC-827 cell lines transfected with miR-4739 mimic or negative control. e and $f$ The mRNA and protein levels of PCSK9 was examined by qRT-PCR or western blot in the NCl-H1975 and Clau-3 cell lines transfected with circRUNX1 or mock control. $g$ and $h$ The mRNA and protein levels of PCSK9 was examined by qRT-PCR or western blot in the PC-9 and HCC-827 cell lines transfected with circRUNX1 shRNA or negative control. $i$ and $j$ The mRNA and protein levels of PCSK9 was examined by qRT-PCR or western blot in the NCl-H1975 and Clau-3 cell lines transfected with mutant circRUNX1 or mock control. Data are presented as the means \pm SD of three independent experiments. ${ }^{\star *} P<0.01,{ }^{*} * \mathrm{P}<0.001$. NS, no significant. 
$\mathrm{a}$

PCSK9

Tubulin b

PCSK9

Tubulin

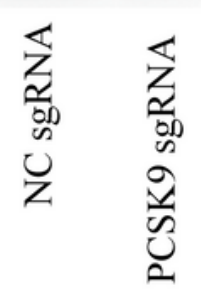

C $12007-0$ Mock IgG

$\approx$ circRUNX1 IgG

畐 $800-7$ circRUNX1 PD-1 mAb

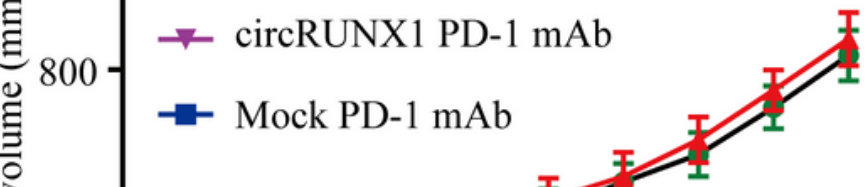

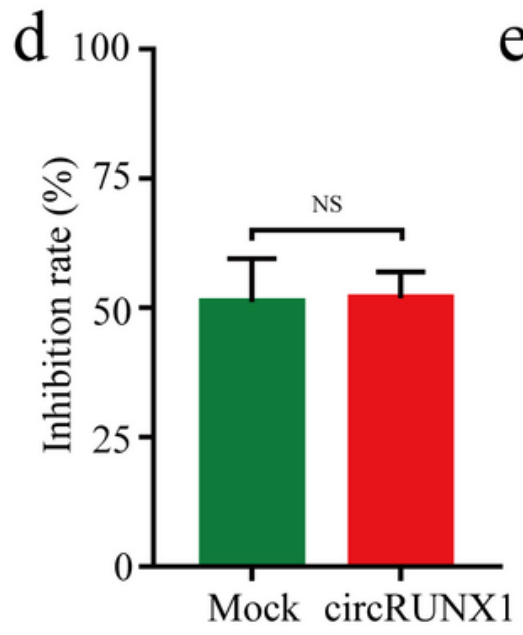

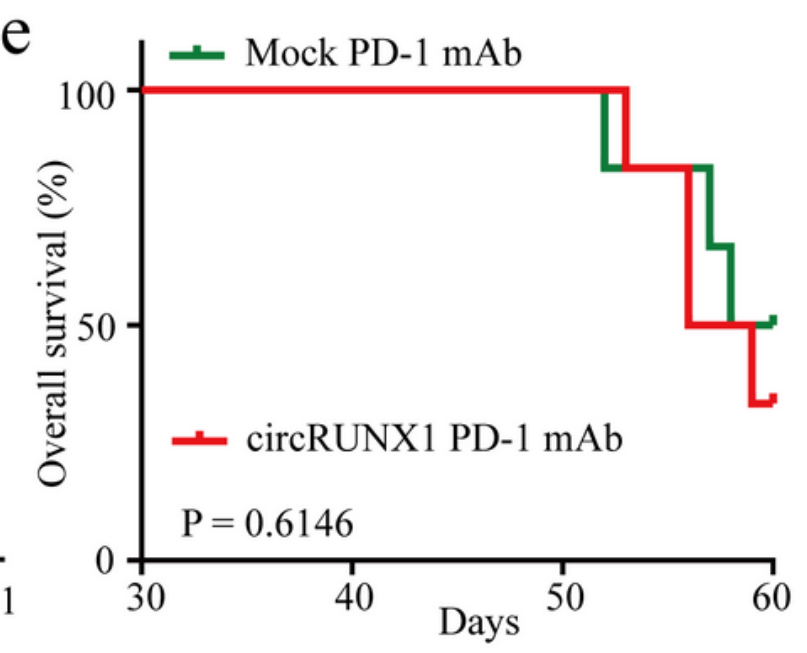

f

g
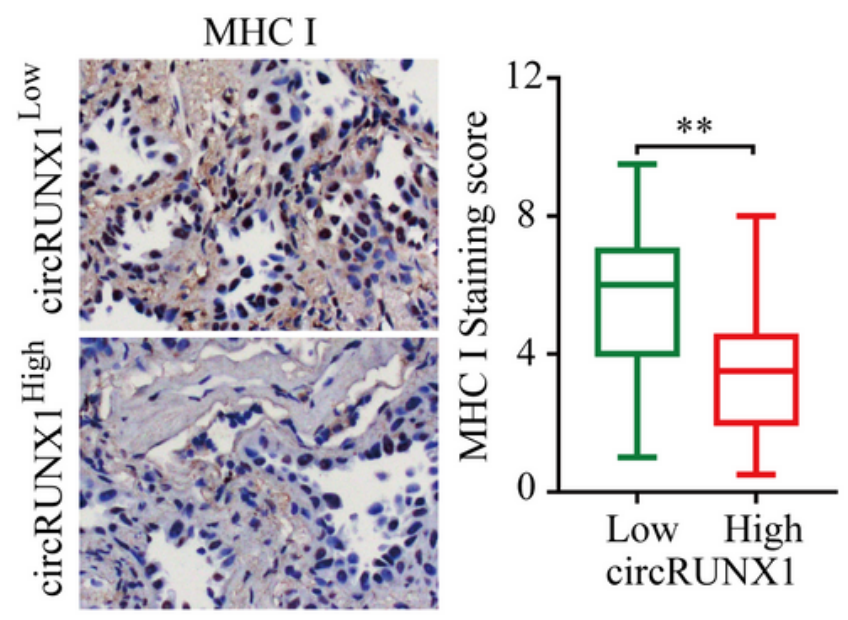

$\mathrm{h}$

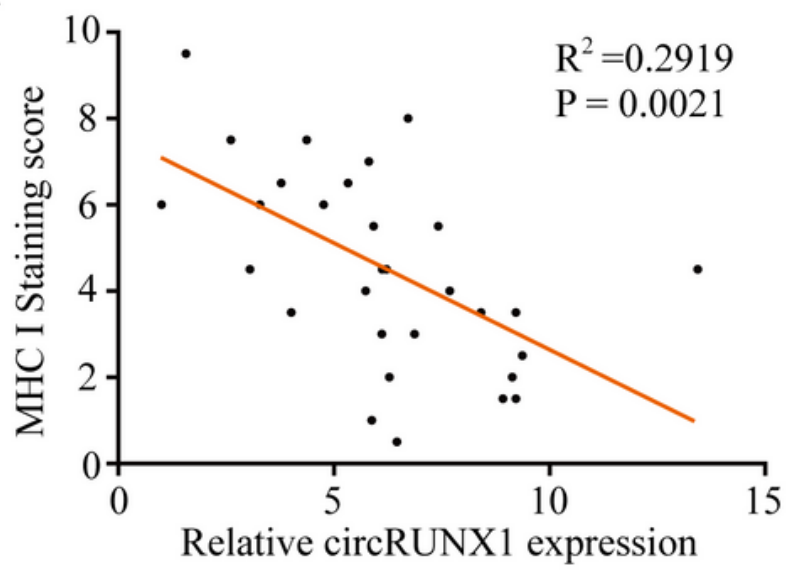

Figure 6

Knocking out PCSK9 reverses the cancer-promoting function of circRUNX1 in the LUAD. a PCSK9 expression in $\mathrm{NCl}-\mathrm{H} 1975$ cells was detected by western blot. b PCSK9 expression in $\mathrm{NCl}-\mathrm{H} 1975$ cells with PCSK9 knocking out was modified by lentivirus-mediated circRUNX1transfection. c NCl-H1975-KOP-Mock or NCl-H1975-KOP-circRUNX1 cells were subcutaneously injected into huNSG mice, and when tumors volume reached about $100 \mathrm{~mm}$, and mice were treated with IgG or PD-1 antibody (The data are 
presented as the mean $\pm S D ; n=6$ ). $d$ The data are expressed as the inhibition rate of tumors. $e$ Comparison of the OS curves for mice with forced circRUNX1 expression or Mock control in xenograft LUAD tumors without PCSK9 expression that were treated with PD-1 antibody. $\mathrm{f} \mathrm{MHC} \mathrm{I-positive} \mathrm{cells} \mathrm{in} \mathrm{the}$ LUAD tissues were analyzed by IHC. g A positive correlation between circRUNX1 and MHC I expression was detected in tumor tissues from LUAD patients $(R 2=0.2919 ; \mathrm{P}=0.0021) .{ }^{*} \mathrm{P}<0.01$.

\section{Supplementary Files}

This is a list of supplementary files associated with this preprint. Click to download.

- SupplementaryFiguresandFigurelegends.doc

- SupplementaryMethods.docx

- SupplementaryTables.doc 